\title{
La Heterogeneidad Ambiental en un Matorral Xerófilo
}

\author{
Gabriela Santibáñez-Andrade ${ }^{1,3}$, Silvia Castillo-Argüero ${ }^{1}$, José Alejandro \\ Zavala-Hurtado ${ }^{2}$, Yuriana Martínez Orea ${ }^{1}$ y Mariana Hernández Apolinar ${ }^{1}$ \\ 'Departamento de Ecología y Recursos Naturales, Facultad de Ciencias, \\ Universidad Nacional Autónoma de México 04510. \\ ${ }^{2}$ Universidad Autónoma Metropolitana, D. F. México. \\ ${ }^{3}$ Autor para la correspondencia. Correo-e: gabysant@ciencias.unam.mx
}

\begin{abstract}
Resumen: Se caracterizó la heterogeneidad biótica y abiótica tanto espacial como temporal en dos zonas del matorral xerófilo de la Reserva Ecológica del Pedregal de San Ángel (REPSA). Se identificaron nueve grupos florísticos, los cuales presentaron una distribución diferencial de las especies vegetales presentes en la zona. Cada uno de los grupos fue nombrado respecto a las especies indicadoras, las cuáles fueron consideradas como determinantes de la fisonomía de la comunidad. Utilizando los análisis multivariados (TWINSPAN y CANOCO) se observó que la composición de especies de los grupos se modifica espacial y temporalmente. Este estudio sugiere que la variación de algunos factores abióticos como la profundidad del suelo y la pendiente influye de diferente manera en la heterogeneidad del sistema. La heterogeneidad temporal fue evidente al existir una diferente composición florística en la zona a lo largo de la temporada de lluvias y la temporada de secas.
\end{abstract}

Palabras clave: heterogeneidad, microambiente, matorral xerófilo, análisis multivariados

\begin{abstract}
Biotic and abiotic heterogeneity in spatial and temporal scales were characterized for two areas of the xerophytic shrubland of the "Pedregal de San Ángel" (REPSA) ecological reserve. Nine floristic groups were identified and they showed a differential distribution of the plant species that inhabit this community. Each one of the groups was named according to the key species, therefore they were considered as determinants in the communities physiognomy. Through the use of multivariate analysis (TWINSPAN and CANOCO) we observed that species composition in the groups is modified in spatial and temporal scales. This study suggests that variation of some abiotic factors such as soil depth and slope influences differentially the systems heterogeneity. Temporal heterogeneity was evident since there was a different floristic composition in this area during the dry and the rainy season.
\end{abstract}

Key words: heterogeneity, microenvironment, xerophytic shrubland, multivariate analysis

$\mathbf{L}^{2}$ a heterogeneidad ambiental resulta de la variación de los factores abióticos que a su vez puede verse reflejada en los patrones de distribución y abundancia de las especies vegetales (Stewart et al., 2000). Esto debido a que la variación espacial y temporal en el ambiente influye en el establecimiento, en su desarrollo, reproducción y supervivencia de las plantas. Esta heterogeneidad espacial y temporal es una de las principales características de las zonas áridas y semiáridas (Breshears et al., 1998), en los cuáles la estacionalidad es uno de los factores causantes de la heterogeneidad temporal, cuya diferencias condicionan la coexistencia de las especies vegetales, pues la presencia, duración e intensidad de las precipitaciones se vuelve un recurso limitante (Tongway et al., 2004). Asimismo, la competencia por recursos entre dos o más especies se modifica con la variación estacional, pues cambia la disponibilidad de éstos, creando una separación temporal para las especies y con ello evitar la competencia por interferencia (Chesson y Huntly, 1985; Rosenberg y Freedman, 1994; Escribano et al., 2008).

Algunos autores sostienen que la formación de mosaicos ambientales está determinada por la variación topográfica y edáfica, señalando que existe una fuerte relación entre el relieve y los patrones de distribución y abundancia de las especies (Lieberman et al., 1996; Titus y Tsuyuzaki, 2003; Xu et al., 2000). Asimismo esta variación topográfica está estrechamente relacionada con la pendiente y profundidad del suelo (Chen et al., 1996; Clark et al., 1998; Paruelo et al., 2005).

La formación de microambientes es resultado de esta variación topográfica. Se ha demostrado que los microambien- 
tes juegan un papel importante en el mantenimiento de la diversidad, debido a que la presencia de una gran variedad de ellos puede crear una amplia gama de condiciones favorables para un mayor número de especies (Clark et al., 1993; Smith y Smith, 2001; García, 2006). Este tipo de respuestas, aunado al desfasamiento en la utilización de los recursos en espacio y tiempo, permite que algunas especies dominen algunos microambientes y en otros sólo se mantengan en un tamaño poblacional mínimo (Guo y Brown, 1996).

Varios han sido los estudios que han descrito la presencia de especies indicadoras, asociadas con un microambiente y a sus condiciones microclimáticas, las cuales a menudo representan el mayor componente dentro del sistema o subsistemas y se asume que controlan la estructura de la comunidad en espacio y tiempo (Menge et al., 1994; Tanner et al., 1994; Lindenmayer et al., 2000). Estas especies en general presentan en su distribución y abundancia una correlación con ciertos intervalos de un determinado factor abiótico, como sugieren las leyes de tolerancia y del mínimo (Becker et al., 1998; Simberloff, 1998).

En este estudio se realizó una caracterización de la heterogeneidad ambiental, se determinó si existen grupos florísticos en función de algunos factores físicos y se discutieron los cambios en la estructura y su relación con la heterogeneidad temporal de un matorral xerófilo.

\section{Materiales y Métodos}

Sitio de estudio. El presente estudio se llevó a cabo en la Reserva Ecológica "Pedregal de San Ángel" (REPSA) la cual se localiza al sur del Distrito Federal (Delegación Coyoacán) entre las coordenadas $19^{\circ} 18^{\prime} 21^{\prime \prime}-19^{\circ} 20^{\prime} 11^{\prime \prime} \mathrm{N}$ y $99^{\circ} 10^{\prime} 15^{\prime \prime}-99^{\circ} 12^{\prime} 4^{\prime \prime} \mathrm{O}$ en un intervalo altitudinal de
2,292 a 2,365 m s.n.m (figura 1). El clima es templado subhúmedo, con una precipitación promedio anual de $833 \mathrm{~mm}$ y una temperatura media anual de $15.6^{\circ} \mathrm{C}$ (Castillo-Argüero et al., 2007). Presenta una marcada estacionalidad, siendo la época de lluvias de junio a octubre y la de secas de noviembre a mayo. El sustrato es de roca basáltica originado del derrame del volcán Xitle, por lo tanto, el suelo es escaso, poco profundo y rocoso (Rzedowski, 1954). El enfriamiento desigual de la lava originó una heterogeneidad geomorfológica, dando lugar a microambientes con diversas condiciones de suelo, humedad, temperatura y exposición a la luz (Peralta y Prado, 2009). Entre los tipos de microambientes se encuentran: a) planos, sitios continuos de roca con diferentes grados de inclinación; b) oquedades, sitios con presencia de concavidades, suelen ser poco profundas; c) grietas, zonas de fisura y fractura en la roca angostas y profundas; d) hondonadas, sitios de depresión cóncavas de poco a muy profundas las cuáles pueden ser grandes e incluir otros microambientes y e) promontorios, sitios de roca salientes que les confieren mayor altura que el resto de los microambientes adyacentes (Cano-Santana, 1994; CastilloArgüero et al., 2007; Santibáñez-Andrade, 2005). El tipo de vegetación que alberga la REPSA corresponde a un matorral xerófilo denominado Senecionetum praecosis, debido a la abundancia de la especie Pittocaulon praecox (= Senecio praecox) (Rzedowski, 1954). Actualmente existe una gran riqueza florística de aproximadamente 377 especies, cuya organización da lugar a una estructura heterogénea (Casti1lo-Argüero et al., 2009). Los estratos presentes en esta zona son: el arbustivo (30 $\mathrm{cm}$ a $1.5 \mathrm{~m}$ de altura), el herbáceo (< $30 \mathrm{~cm})$, el rastrero $(<10 \mathrm{~cm})$ y el arbóreo $(>1.5 \mathrm{~m})$, siendo éste último poco representativo (Rzedowski, 1954; CanoSantana, 1994).

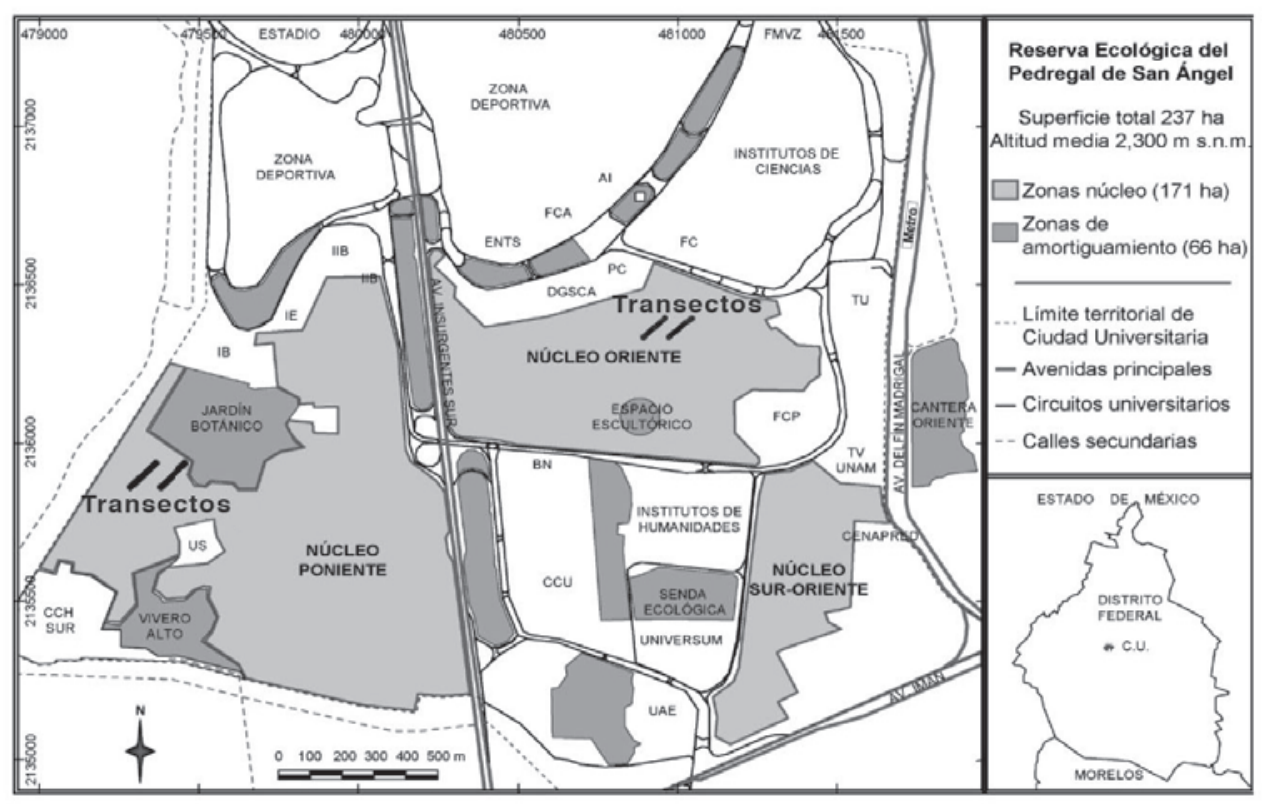

Figura 1. Localización de la Reserva del Pedregal de San Ángel (Gaceta UNAM, 2005). 
Método de campo y análisis de la información. La variación espacial de los factores bióticos y abióticos a escala microambiental fue caracterizada en dos zonas al interior de la REPSA durante la temporada de lluvias (julio y agosto del 2002) y la temporada de secas (marzo y abril del 2003). Con este propósito, se trazaron dos transectos de $100 \times 1 \mathrm{~m}$ en dirección norte-sur en cada una de las zonas, los cuales se dividieron en parcelas de $2 \times 1 \mathrm{~m}$ resultando un total de 200 áreas de muestreo.

La heterogeneidad biótica fue evaluada en función de las especies registrando datos de densidad, cobertura y frecuencia relativa en ambas temporadas. Se registraron los dos diámetros de las proyecciones aéreas de las especies para posteriormente calcular la cobertura adaptando los datos en la fórmula del área del círculo. A partir de los datos estructurales de las especies se determinó su valor de importancia $(V I=$ densidad relativa + cobertura relativa + frecuencia relativa) siguiendo el criterio de van der Maarel (Jongman et al., 1987; Hill, 1979). Con el valor de importancia se realizó un Análisis de Especies Indicadoras de Dos Vías (TWINSPAN) para conocer la existencia de grupos florísticos, utilizando el programa PC-ORD 5.10 (McCune y Mefford, 2006). Se determinaron las especies indicadoras de cada uno de los grupos obtenidos. La clasificación base fue la obtenida con las especies existentes en la temporada de lluvias pues fue la más representativa temporalmente ya que cuenta con una mayor presencia de especies. La clasificación obtenida fue evaluada mediante un análisis discriminante múltiple bajo el criterio de lambda de Wilks, utilizando el programa estadístico SPSS 12 (Tatsuoka, 1971; SPSS, 2003).

La heterogeneidad abiótica fue evaluada en relación a la variación en altitud, pendiente y profundidad de suelo. Los datos de altitud para cada metro del transecto se obtuvieron con ayuda de un teodolito, un GPS y un estadal, para elaborar un perfil topográfico. La pendiente se calculó con los datos de la altitud, el perfil topográfico y la fórmula de la pendiente entre cada metro del transecto. La profundidad del suelo se obtuvo con una varilla calibrada introduciéndola en cada punto. Con estos datos se elaboraron perfiles de variación en cada punto del transecto.

Relación entre los grupos florísticos y los factores abióticos. Para examinar las diferencias entre los grupos florísticos en función de sus variables abióticas se realizó un análisis de varianza (ANDEVA) de una vía con una prueba de Tukey HSD en el programa Statistica 6.0 (Statsoft INC, 2001). Por otro lado, se analizó la relación de los grupos florísticos con los microambientes realizando una correlación de Spearman, la cual es una medida de regresión lineal entre dos variables con valores en escala ordinal, con el programa Statistica 6.0 (Statsoft INC, 2001). Por último, se realizó un Análisis Canónico de Correspondencia (CCA) que permite interpretar la relación ambiente-organismos en ambas temporadas y conocer si las variables abióticas registradas son explicativas y a qué grado, utilizando el programa PC-ORD 5.10 (McCune y Mefford, 2006).

\section{Resultados}

Heterogeneidad biótica. Se registró un total 101 especies, las cuales corresponden al $27 \%$ de las 377 reportadas para la REPSA. Las especies se distribuyeron en 48 familias que corresponden a $66 \%$ de las 73 familias que se tienen registradas para el área. Durante la temporada de secas se observó que la cobertura vegetal se redujo un $45 \%$ de la registrada para la época de lluvias $\left(979.9 \mathrm{~m}^{2}\right.$ de cobertura en lluvias y $444.3 \mathrm{~m}^{2}$ en secas). El análisis de clasificación (TWINSPAN) que se realizó con 85 especies presentes en la temporada de lluvias distinguió nueve grupos florísticos con diferentes especies (figura 2). Cada grupo fue denominado de acuerdo a la especie de mayor VI es decir, Grupo 1) Pittocaulon praecox; Grupo 2) Cheilanthes myriophylla; Grupo 3) Arracacia tolucensis; Grupo 4) Erianthus repens; Grupo 5) Buddleja cordata; Grupo 6) Buddleja cordata y Pittocaulon praecox; Grupo 7) Ageratina petiolaris; Grupo 8) Muhlenbergia robusta y Grupo 9) Wigandia urens.

En el análisis de clasificación para la temporada de secas sólo 25 especies estuvieron presentes. Se encontraron diferencias entre presencia y dominancia de las especies en los grupos, sin embargo las especies indicadoras de cada grupo se mantuvieron con los valores más altos de VI al igual que en la temporada de lluvias.

En la Tabla I se muestran las especies indicadoras durante la temporada de lluvias y secas, determinadas a través de su valor de importancia, se señala el número de especies que conforman los grupos florísticos, así como sus intervalos en factores como profundidad de suelo y pendiente y el número de parcelas de cada grupo que se sitúan en cada tipo de microambiente.

El resultado del análisis discriminante para la temporada de lluvias determinó que el $95.5 \%$ de los casos analizados estuvo correctamente clasificado ( $\lambda$ de Wilks $<0.0001$; $p<$ $0.0001)$. En el caso de la estación seca, el análisis discriminante mostró que solamente el $45.7 \%$ de los casos estuvo correctamente clasificado ( $\lambda$ de Wilks $=0.221 ; p<0.0001$ ). Heterogeneidad abiótica. Se encontró una notable variación en la altitud y la profundidad del suelo en ambos transectos de la zona (figura 3 ).

Relación entre los grupos florísticos y los factores abióticos. El análisis de varianza (ANDEVA) de una vía indicó que los grupos florísticos presentan diferencias significativas importantes en sus factores físicos (figura 4). La profundidad de suelo es el factor en el cual se observó la presencia de cuatro grupos con diferencias significativas según la prueba de Tukey $(p<0.05)$. Respecto a la pendiente se encontraron tres grupos con diferencias significativas entre sí.

Microambientes. En la Tabla II se observan los coeficientes de correlación de Spearman entre los grupos y los cinco 
Gabriela SantibáÑEZ-AndRade, ET AL.

Tabla I. Tabla de factores abióticos, microambientes y valores de importancia de las especies características de los grupos florísticos para la temporada de lluvias (LI) y de secas (S). *Se presentan los valores promedio \pm error estándar para los factores abióticos.

\begin{tabular}{|c|c|c|c|c|c|c|c|c|c|c|c|c|c|c|c|c|c|c|}
\hline \multirow{3}{*}{$\begin{array}{l}\text { Número de grupo } \\
\text { Número total de parcelas } \\
\text { Época }\end{array}$} & \multicolumn{2}{|r|}{1} & \multicolumn{2}{|c|}{2} & \multicolumn{2}{|c|}{3} & \multicolumn{2}{|c|}{4} & \multicolumn{2}{|c|}{5} & \multicolumn{2}{|c|}{6} & \multicolumn{2}{|c|}{7} & \multicolumn{2}{|c|}{8} & \multicolumn{2}{|c|}{9} \\
\hline & \multicolumn{2}{|c|}{17} & \multicolumn{2}{|c|}{10} & \multicolumn{2}{|c|}{14} & \multicolumn{2}{|c|}{25} & \multicolumn{2}{|c|}{22} & \multicolumn{2}{|c|}{19} & \multicolumn{2}{|c|}{8} & \multicolumn{2}{|c|}{65} & \multicolumn{2}{|c|}{20} \\
\hline & $\mathrm{LI}$ & $S$ & $\mathrm{LI}$ & $S$ & $\mathrm{LI}$ & $S$ & $\mathrm{LI}$ & $S$ & $\mathrm{LI}$ & $S$ & $\mathrm{LI}$ & $S$ & $\mathrm{LI}$ & $S$ & $\mathrm{LI}$ & $S$ & $\mathrm{LI}$ & $S$ \\
\hline Número total de especies & 46 & 21 & 31 & 10 & 36 & 9 & 55 & 20 & 54 & 19 & 46 & 21 & 27 & 16 & 68 & 25 & 44 & 24 \\
\hline Profundidad de suelo $(\mathrm{cm})$ & 3.2 & \pm 0.6 & $2 \pm$ & 1.2 & & 0.7 & 4.5 & \pm 0.7 & & 0.9 & 4.4 & \pm 0.7 & 3.6 & \pm 0.9 & & 0.5 & 4.9 & \pm 0.8 \\
\hline Luz a un metro (luxos) & 264 & \pm 31.4 & $423 \pm$ & \pm 62.2 & 444 & $=58.3$ & $266=$ & 26.5 & $329=$ & $=38.7$ & 361 & \pm 35.6 & 522 & \pm 90.0 & $297=$ & \pm 22.5 & $397=$ & \pm 41.9 \\
\hline Luz a nivel del suelo (lux.) & 163 & \pm 46.1 & $300 \pm$ & \pm 69.9 & 314 & $=52.9$ & $152=$ & 22.3 & $168=$ & 27.7 & 150 & \pm 24.8 & 309 & \pm 90.7 & $122=$ & \pm 10.6 & $137=$ & \pm 24.8 \\
\hline Pendiente $\left(^{\circ}\right)$ & 17.4 & \pm 3.3 & 19.4 & \pm 4.3 & 8.5 & \pm 2.5 & 10.9 & \pm 1.4 & 10.1 & \pm 2.1 & 21.4 & \pm 3.0 & 20.3 & \pm 4.0 & 14.6 & \pm 1.3 & 16.4 & \pm 6.6 \\
\hline \multicolumn{19}{|c|}{ Número de microambientes por grupo } \\
\hline Plano & \multicolumn{2}{|c|}{5} & \multicolumn{2}{|c|}{7} & \multicolumn{2}{|c|}{6} & & 3 & & 2 & & 2 & & 0 & & 8 & & 6 \\
\hline Hondonada & & 1 & & 0 & & 1 & c & 0 & & 9 & & 14 & & 3 & & 27 & & 10 \\
\hline Promontorio-cima & & 9 & & 1 & & 1 & 1 & 2 & & 1 & & 3 & & 4 & & 9 & & 0 \\
\hline Ноуо & & 2 & & 1 & & 3 & ( & 0 & & 0 & & 0 & & 0 & & 6 & & 3 \\
\hline Grieta & & 0 & & 1 & & 3 & ( & 0 & & 0 & & 0 & & 1 & & 5 & & 1 \\
\hline Especies Indicadoras & & & & & & & & & & & & & & & & & & \\
\hline $\begin{array}{l}\text { Pittocaulon praecox (Cav.) } \\
\text { H.Rob. \& Brettell }\end{array}$ & 16.1 & 13.7 & -- & -- & 2.7 & 2.3 & 2.6 & 1.9 & -- & -- & 9.2 & 7.5 & 1.4 & 1.3 & 1.4 & 1.2 & -- & -- \\
\hline Bursera cuneata Engl. & 3.7 & -- & -- & -- & -- & -- & -- & -- & -- & -- & -- & -- & -- & -- & -- & -- & -- & -- \\
\hline $\begin{array}{l}\text { Cheilanthes myriophylla } \\
\text { Desv. }\end{array}$ & 4.7 & 2.3 & 16.5 & 9.8 & 1.9 & 0.9 & 1.3 & -- & 2.0 & -- & 1.6 & 0.6 & -- & -- & 1.1 & 1.2 & 2.2 & 3.3 \\
\hline Tagetes lunulata Ortega & -- & -- & 9.1 & -- & 3.9 & -- & -- & -- & -- & -- & -- & -- & -- & -- & -- & -- & -- & -- \\
\hline $\begin{array}{l}\text { Sellaginella lepidophylla } \\
\text { (Hook.et Greville) Spring }\end{array}$ & 0.6 & -- & 8.1 & 2.5 & 2.8 & -- & 0.9 & -- & -- & -- & -- & -- & -- & -- & -- & -- & -- & -- \\
\hline $\begin{array}{l}\text { Astrolepis sinuata } \\
\text { (Lag. ex Sw.) D.M. } \\
\text { Benham \& Windham }\end{array}$ & -- & 3.3 & -- & 10.6 & -- & 5.8 & -- & 4.3 & -- & 0.5 & -- & 1.2 & -- & 1.2 & -- & 3.7 & -- & 2.5 \\
\hline Arracacia tolucensis Hemsl. & -- & -- & 0.3 & -- & 9.2 & -- & -- & -- & -- & -- & -- & -- & -- & -- & -- & -- & -- & -- \\
\hline Buddleja cordata Kunth & 1.1 & 1.0 & -- & -- & 7.7 & 6.5 & -- & -- & 26.8 & 26.6 & 19.9 & 19.8 & -- & -- & 2.7 & 2.2 & -- & -- \\
\hline Erianthus repens P.Beauv. & 1.2 & 3.3 & -- & -- & -- & -- & 13.0 & 12.2 & 1.2 & -- & -- & 2.6 & -- & 2.0 & 3.9 & 4.2 & -- & 3.2 \\
\hline $\begin{array}{l}\text { Manfreda scabra (Ort.) } \\
\text { McVaugh }\end{array}$ & -- & -- & -- & -- & -- & -- & 5.9 & -- & -- & -- & -- & -- & -- & -- & 2.3 & -- & -- & -- \\
\hline $\begin{array}{l}\text { Iresine difusa Humb. Et } \\
\text { Bonpl. Ex Schult.) Moq. }\end{array}$ & -- & -- & -- & -- & -- & -- & -- & -- & -- & 6.9 & 0.4 & 1.3 & 2.2 & -- & 0.3 & 1.2 & 0.8 & 1.5 \\
\hline Montanoa tomentosa Cerv. & -- & 1.1 & -- & -- & -- & -- & -- & -- & -- & -- & 5.6 & 7.3 & -- & 2.2 & -- & 1.6 & 4.5 & 2.9 \\
\hline Salvia mexicana L. & 2.7 & 2.6 & -- & -- & -- & -- & -- & -- & -- & -- & 6.2 & 5.7 & 3.0 & 2.9 & 1.0 & 1.2 & 8.5 & 4.3 \\
\hline $\begin{array}{l}\text { Ageratina petiolaris } \\
\text { (Moc. \& Sessé ex DC.) } \\
\text { R.M.King \& H.Rob. }\end{array}$ & 1.8 & 1.4 & -- & -- & -- & -- & -- & -- & -- & -- & 6.4 & 6.0 & 10.0 & 8.0 & -- & -- & 1.3 & 1.0 \\
\hline Verbesina virgata Cav. & 1.4 & 1.8 & -- & -- & -- & -- & -- & 1.2 & 3.0 & 2.9 & 2.5 & 1.8 & 9.3 & 8.5 & 9.7 & 7.0 & 2.7 & 1.9 \\
\hline Stevia ovata Dum.Cours. & -- & 2.6 & -- & -- & -- & -- & -- & -- & 2.7 & 0.9 & -- & -- & 6.6 & -- & 0.9 & -- & -- & 1.0 \\
\hline $\begin{array}{l}\text { Muhlenbergia robusta } \\
\text { (E.Fourn.) Hitchcock }\end{array}$ & -- & -- & -- & -- & -- & -- & 3.2 & 2.7 & -- & -- & 2.5 & 2.0 & -- & -- & 12.7 & 10.9 & 3.7 & 3.5 \\
\hline Wigandia urens Kunth & -- & -- & -- & -- & -- & -- & 1.7 & 1.2 & 0.9 & 1.0 & -- & -- & -- & -- & 1.3 & 1.8 & 9.9 & 7.3 \\
\hline
\end{tabular}

Tabla II. Coeficientes de correlación de Spearman de los grupos florísticos con los microambientes de la REPSA. Se presentan en negritas los valores más significativos $(\mathrm{p}<0.05)$

\begin{tabular}{lccccccccc}
\hline Microambiente & Grupo 1 & Grupo 2 & Grupo 3 & Grupo 4 & Grupo 5 & Grupo 6 & Grupo 7 & Grupo 8 & Grupo 9 \\
\hline Plano & -0.01 & $\mathbf{0 . 2 0}$ & 0.08 & $\mathbf{0 . 1 8}$ & -0.15 & -0.14 & -0.13 & -0.04 & 0.01 \\
Hondonada & -0.19 & -0.17 & -0.17 & -0.29 & $\mathbf{0 . 3 7}$ & $\mathbf{0 . 2 2}$ & 0.01 & 0.06 & $\mathbf{0 . 1 3}$ \\
Promontorio & $\mathbf{0 . 2 5}$ & -0.05 & -0.08 & $\mathbf{0 . 2 6}$ & -0.17 & 0.01 & $\mathbf{0 . 1 5}$ & -0.10 & -0.01 \\
Oquedad & 0.04 & 0.02 & $\mathbf{0 . 1 4}$ & -0.10 & -0.09 & -0.09 & -0.05 & 0.04 & 0.09 \\
Grieta & -0.07 & 0.04 & $\mathbf{0 . 1 9}$ & -0.09 & -0.08 & -0.08 & $0-06$ & 0.06 & -0.01 \\
\hline
\end{tabular}




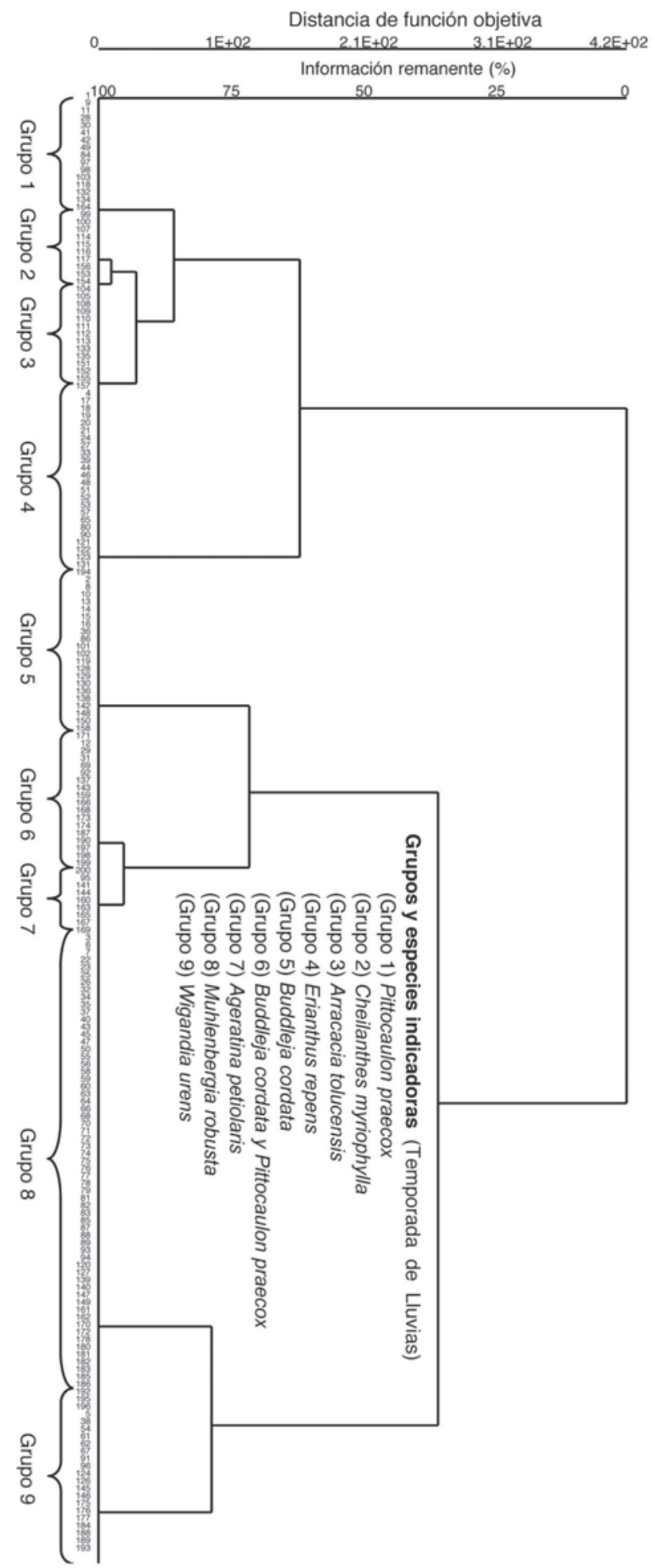

Figura 2. Dendrograma de nueve grupos florísticos distinguidos por el Análisis de Especies Indicadoras de Dos Vías TWINSPAN de un total de 200 parcelas en la REPSA. En el recuadro se muestran las especies características de cada grupo de la temporada de lluvias. tipos de microambientes. Se encontró que cada uno de los grupos está relacionado con varios tipos de microambientes. Los grupos 1) Pittocaulon praecox, 4) Erianthus repens y 7) Ageratina petiolaris, se encuentran más en sitios con promontorios. El grupo 2) Cheilanthes myriophilla se ubica más en planos que en cualquier otro microambiente mientras que el grupo 3) Arracacia tolucensis se distribuye en grietas y oquedades. El grupo 5) Buddleja cordata, el grupo 6) Buddleja cordata y Pittocaulon praecox y el grupo 9) Wigandia urens se establecen más en las hondonadas. Sin embargo el grupo 8) Muhlenbergia robusta no presentan alguna afinidad clara con los distintos tipos de microambientes.

La figura 5a muestra la ordenación de los nueve grupos florísticos, las especies y los factores ambientales de la temporada de lluvias obtenida mediante el Análisis de Correspondencia Canónica (CCA). La ubicación de los grupos señala que la variación en la pendiente tiene un efecto significativo en las especies de los grupos 6) Buddleja cordata, 7) Ageratina petiolaris y 9) Wigandia urens, afectando débilmente a los grupos 1) Pittocaulon praecox y 2) Cheilanthes myriophylla. Por otro lado, la variación en la profundidad del suelo tiene un efecto más directo en la presencia de especies de los grupos 4) Erianthus repens, 5) Buddleja cordata y 8) Muhlenbergia robusta, sin embargo el grupo 3) Arracacia tolucensis presenta una débil relación con éste factor. Cabe mencionar que los grupos 8 y 9 por su cercanía a ambos vectores pueden estar influenciados por ambos gradientes.

Los valores de las raíces características para los dos primeros ejes de la ordenación para la temporada de lluvias (0.61 y 0.35$)$ sugieren que existe una separación entre los grupos florísticos asociada a gradientes ambientales a lo largo de estos ejes de variación. La correlación entre las variables ambientales y la presencia de especies para los tres ejes fue significativa, además de que se registró un total de $34.6 \%$ de la varianza explicada por los datos de las especies.

La figura 5 b muestra la ordenación de los nueve grupos florísticos, las especies y los factores ambientales de la temporada de secas obtenida mediante el CCA. Los grupos 4) Erianthus repens, 5) Buddleja cordata y 8) Muhlenbergia robusta, se encuentran relacionados con la profundidad del suelo, también el grupo 3) Arracacia tolucensis se encuentra relacionado a este factor aunque más débilmente al igual que ocurrió en la temporada de lluvias. Los grupos 1) Pittocaulon praecox, 6) Buddleja cordata, 7) Ageratina petiolaris y 9) Wigandia urens están cercanamente relacionados con la pendiente, por su lado el grupo 2 ) Cheilanthes myriophylla también se encuentra afectado por la variación de la pendiente pero en menor grado. Estos resultados son similares a los encontrados en la temporada de lluvias. Sin embargo en esta temporada la ubicación de los grupos 6 y 9 señala que podrían estar afectados por ambos gradientes.

Durante la temporada de secas los valores de las raíces características para los dos primeros ejes de la ordenación 

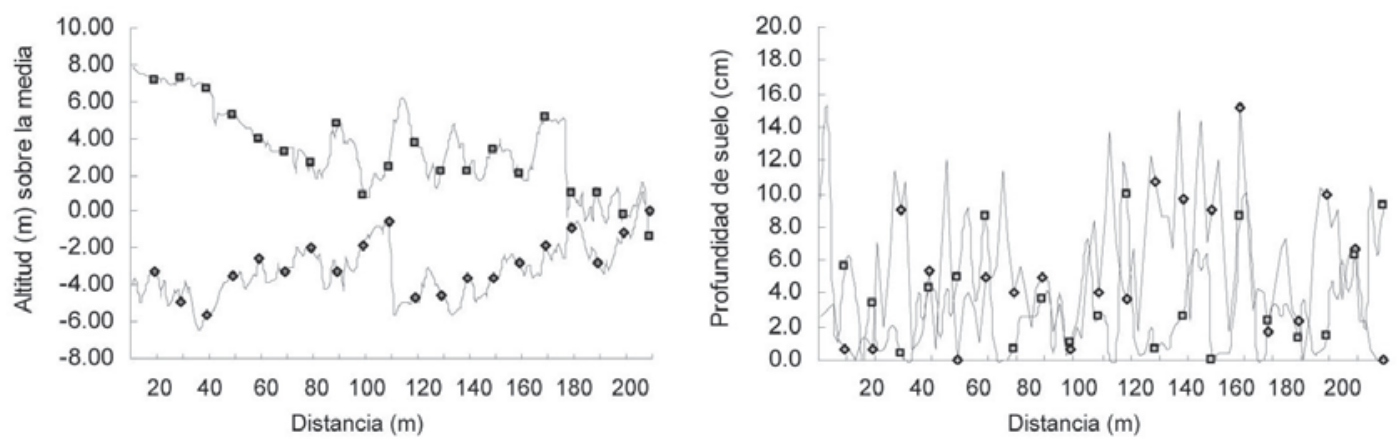

Figura 3. Variación de los factores físicos en los transectos de la REPSA. Los valores de altitud son relativos a la media. Transecto 1 y 2 de Facultad de Ciencias $\downarrow$. Transecto 2 y 3 de Jardín Botánico

(0.230 y 0.139$)$ en este caso indican que no existe una separación tan clara entre los grupos florísticos asociados a gradientes ambientales como se observó en la temporada de lluvias. Sin embargo, la correlación entre las variables ambientales y la presencia de especies para los tres ejes resultó significativa y se registró un total de $37 \%$ de la varianza explicada por los datos de las especies.

Los datos aleatorizados generados con permutaciones Monte Carlo indican que tanto las raíces características para los dos primeros ejes, como la correlación entre las especies, las variables ambientales y los dos ejes de la ordenación, son significativas $(p<0.05)$, lo que sugiere que los resultados obtenidos con el CCA no se deben al azar y el diagrama de ordenación proporciona una representación significativa de la distribución de las especies y las variables ambientales estudiadas.

\section{Discusión y conclusiones}

En varios estudios frecuentemente se hace referencia a la existencia de grupos ecológicamente disímiles, en cuanto a la forma en la que las especies aprovechan los recursos y su clasificación se basa principalmente en las condiciones microambientales en la que las especies se establecen. El clasificar a las especies en grupos o gremios relativamente homogéneos es importante porque permite conformar patrones generales y hacer predicciones acerca de procesos a nivel de comunidad en cualquier sitio de estudio (Swaine y Whitmore, 1988). La identificación de nueve agrupaciones florísticas en la REPSA indicó la existencia de "gremios" de especies que posiblemente presenten requerimientos ambientales semejantes como lo mencionan Finegan en 1993 (in Louman et al., 2001) y Root (1967). Las especies indicadoras de cada grupo fueron aquellas que presentaron un mayor valor de importancia en cada grupo, reflejando que en aquellas parcelas existen condiciones más adecuadas para la especie, aun cuando se encontraban con una valor de importancia menor en otros grupos (Finegan, 1993). Es importante mencionar que en este estudio algunas de las especies consideradas características como Pittocaulon praecox, Buddleja cordata, Muhlenbergia robusta, Wigandia urens y Erianthus repens, anteriormente ya habían sido consideradas como verdaderas dominantes estructurales y fisonómicas del matorral xerófilo en cuestión (Meave et al., 1994). Este resultado es interesante y puede indicar que estas especies desempeñan un papel importante en la dinámica de la comunidad, afectando la disponibilidad de recursos para sus especies acompañantes y creando a su vez una mayor
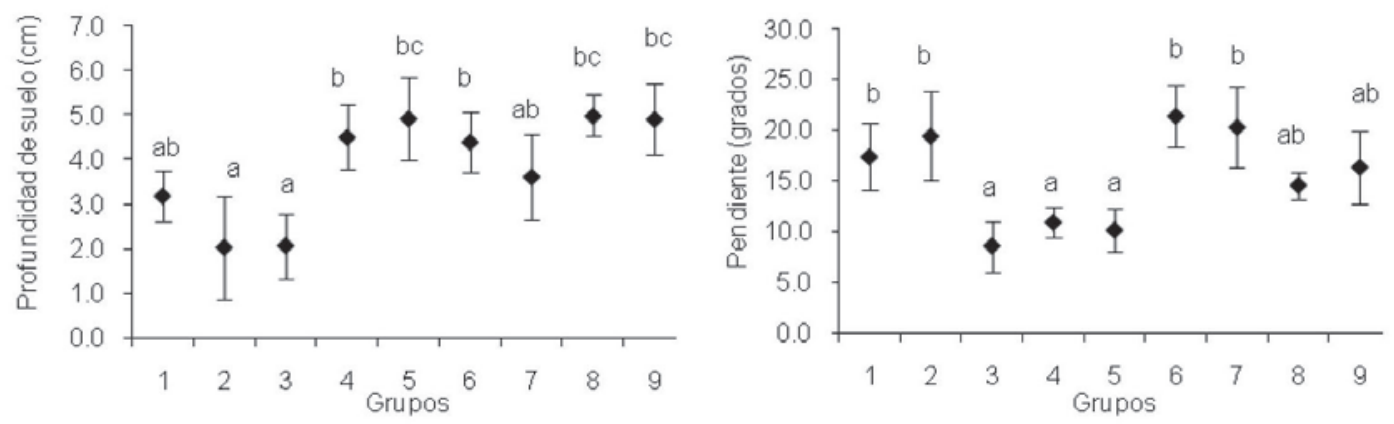

Figura 4. Comparación de los factores físicos entre los grupos florísticos. Los intervalos indican \pm E.E. error estándar. y el punto central representa la media. Se observan diferencias significativas entre los grupos en las dos variables ANDEVA de una vía. Los grupos con la misma letra no difieren significativamente Tukey $(p<0.05)$. 

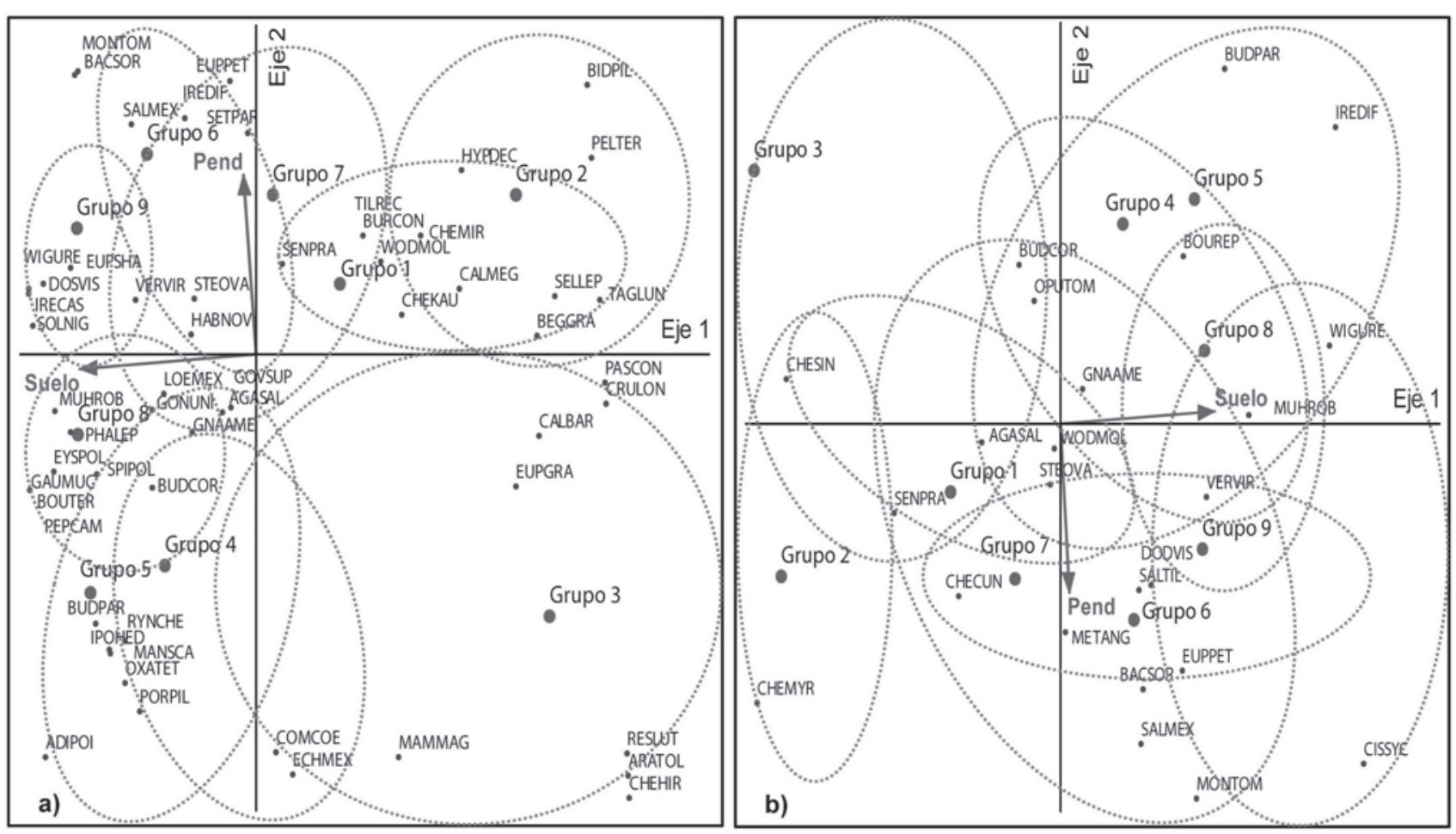

Figura 5. Análisis de correspondencia canónica de nueve grupos florísticos y dos variables ambientales correspondiente a la temporada de lluvias (a) y temporada de secas (b).

heterogeneidad abiótica (Pickett y Rogers, 1997; Shiyomi y Yoshimura, 2000), partiendo del hecho de que la respuesta de las especies a la presencia de la heterogeneidad ambiental depende de muchos factores, entre ellos, la escala de su ambiente, sus requerimientos ambientales e inclusive la presencia de otras especies (Wiens, 1989).

Por otro lado, la frecuencia de la mayoría de las especies en los grupos florísticos se registró de manera diferencial (ver tabla I), en general como consecuencia de algunas de sus características como su tamaño, forma de vida, ciclo de vida, patrones fenológicos y sus requerimientos ambientales (Hutchings y De Kroon, 1994; Rosenberg y Freedman, 1994). Por otro lado, se observó que existen especies que son muy susceptibles a la variación microambiental y especies que no son afectadas. Estos resultados indican que la variedad de condiciones microambientales permite que distintas formas de vida y tipos de crecimiento puedan coexistir en la REPSA (Castillo-Argüero et al., 2004; Swaine y Whitmore, 1988).

De acuerdo con los resultados obtenidos, la REPSA cuenta con un alto grado de heterogeneidad debido en gran parte a su topografía accidentada y la existencia de varios tipos de microambientes (Santibáñez-Andrade, 2005). Es posible apreciar en la figura 4 que la mayoría de los grupos florísticos presentan diferencias en sus condiciones ambientales, ya que, aunque algunos sean similares en cuanto a su presencia en cierto intervalo de profundidad de suelo, en el factor pendiente difieren significativamente. Esto es un indicador de la variedad de condiciones que una vez combinadas generan una diversidad de microambientes en los cuales es posible el establecimiento de un mayor número de especies.

En relación a los grupos florísticos y los microambientes, se observó que al analizar su relación éstos fueron, en general, determinantes en la distribución de dichas agrupaciones. Las especies indicadoras pueden estar relacionadas con algunos tipos de microambientes lo cual depende una vez más de sus requerimientos ambientales. En el caso del grupo 8 caracterizado por Muhlenbergia robusta se observa que es el grupo de más amplia distribución en diferentes microambientes y no presentó ninguna afinidad específica lo cual pueda deberse a las características de la especie o que intervienen otros factores asociados a su distribución.

Se observaron cambios en la presencia y dominancia de especies debido a la estacionalidad. La reducción de la cobertura vegetal que se llega a apreciar en la temporada de secas indica que existen condiciones ambientales distintas asociadas a la temporalidad, las cuales también influyen en la disminución del valor de importancia relativa de las especies en la zona de la REPSA. La mayoría de las especies "características" de los grupos durante la temporada de lluvias permanecieron con altos valores de importancia (VI) 
durante la temporada de secas. Un hecho que se observó en este estudio fue, que en la temporada de lluvias, existe una mayor cantidad de especies, lo cual hace que la distinción de los grupos florísticos sea más evidente, pero durante la temporada de secas las condiciones ambientales se modifican, disminuye la cantidad de especies y es más probable que los grupos florísticos compartan especies entre sí. Por lo tanto, las diferencias entre los grupos se hacen más pequeñas y no permiten una especialización de las especies a un ambiente determinado como ocurría en la temporada de lluvias (Swaine y Whitmore, 1988).

Relación entre los grupos florísticos y la variación de los factores ambientales. Al realizar la ordenación directa se observó que las variables ambientales de profundidad de suelo y pendiente influyen de diferente manera en la distribución de las especies en los grupos florísticos (Asada, 2002). Por ejemplo, durante la temporada de lluvias, la variación en el suelo afecta principalmente a las asociaciones de Erianthus repens (Grupo 4), Buddleja cordata (Grupo 5) y Muhlenbergia robusta (Grupo 8) debido a que la escasa cantidad de suelo, en algunos casos no ofrece una superficie suficiente para el sostenimiento de especies leñosas o de gran tamaño. Por otro lado, la cantidad de nutrientes y sales minerales necesarias para el desarrollo de las plantas es mínima en un suelo de volumen reducido (Rzedowski, 1954). La variación en la pendiente, por otro lado, afecta también en gran parte la tasa de acumulación de suelo, los gradientes de temperatura y la humedad relativa en las parcelas (Chen et al., 1999). Estas variaciones tienen un efecto principalmente en las asociaciones de Pittocaulon praecox (Grupo 6), Ageratina petiolaris (Grupo 7) y Wigandia urens (Grupo 9). Esto indica que las fluctuaciones espaciales en los factores abióticos influyen directamente en la estructura de la comunidad y las diferencias microclimáticas determinan la distribución de las especies en los diferentes grupos. Así, la alta diversidad de la REPSA puede ser explicada por las respuestas individuales de las especies a los efectos de los factores abióticos como diferencias de profundidad del suelo y la pendiente, además de los efectos de la presencia de otras especies.

\section{Agradecimientos}

Biol. Marco Antonio Romero Romero por la elaboración de tablas y figuras, al M. en C. Oswaldo Nuñez Castillo y Dra. Patricia Guadarrama Chávez por su apoyo en el trabajo de campo y al proyecto PAPIME DO203398 por el apoyo otorgado.

\section{Literatura Citada}

Asada T. 2002. Vegetation gradients in relation to temporal fluctuation of environmental factors in Bekanbeushi peatland, Hokkaido, Japan. Ecological Research 17:505-518.

Becker B., Terrones F.M. y Horchler P. 1998. Especies indicadoras de la flora acompañante en campos de cultivo de los Andes. <www.condesan.org/memoria/Docsagrobiod.htm> (Consultado 12 octubre 2009)

Breshears, D. D., Nyhan, J. W., Heil, C. E. y Wilcox, B. P. 1998. Effects of woody plants on microclimate in a semiarid woodland: Soil temperature and evaporation in canopy and intercanopy patches. International Journal of Plants Sciences 159:1010-1017.

Cano-Santana, Z. 1994. Flujo de energía a través de Sphenarium purpurascens (Orthoptera: Acrididae) y productividad primaria neta aérea en una comunidad xerófita. Tesis Doctoral, Facultad de Ciencias, Universidad Nacional Autónoma de México, México, D.F. 198 pp.

Castillo-Argüero S., Martínez-Orea, Y., Romero-Romero, M. A., Guadarrama-Chávez, P., Núñez-Castillo, O., Sánchez-Gallén, I. y Meave, J.A. 2007. La Reserva Ecológica del Pedregal de San Ángel. Aspectos florísticos y ecológicos. Universidad Nacional Autónoma de México. México. D.F.

Castillo-Argüero S., Montes-Cartas G., Romero-Romero M., Martínez-Orea Y., Guadarrama-Chávez P., Sánchez-Gallén I. y Nuñez-Castillo O. 2004. Dinámica y conservación de la flora del matorral xerófilo de la Reserva Ecológica del Pedregal de San Ángel (D.F., México). Boletín de la Sociedad Botánica de México 74:51-75.

Castillo-Argüero Silvia., Martínez-Orea Y., Meave J.A., Hernández-Apolinar M., Núñez-Castillo O., Santibáñez-Andrade G. y Guadarrama-Chávez P. 2009. Flora: susceptibilidad de la comunidad a la invasión de malezas nativas y exóticas. En: Lot. A. y Cano-Santana Z. Eds. Biodiversidad del ecosistema del Pedregal de San Ángel, pp 107-133. Universidad Nacional Autónoma de México. México, D.F.

Chen J., Franklin J.F. y Lowe S.J. 1996. Comparison of abiotic and structurally defined patch patterns in a hypothetical forest landscape. Conservation Biology 10:854-862.

Chen J, Saunders S. y Thomas R. 1999. Microclimate in forest ecosystem and landscape ecology. BioScience 49:288-297.

Chesson P.L. 1985. Coexistence of competitors in spatially and temporally varying environments: A look at the combined effects of different sorts of variability. Theoretical Population Biology 28:263-287

Clark D.B., Clark D.A. y Read J.M. 1998. Edaphic variation and the mesoscale distribution of tree species in a neotropical forest. Journal of Ecology 86:101-112.

Clark D.B., Clark D. y Rich, P. 1993.Comparative analysis of microhabitat utilization by saplings of nine tree species in neotropical rain forest. Biotropica 25:397-407.

Escribano P., Palacios-Orueta A. y Oyonarte C. 2008. Cuantificación y distribución espacial de los tipos de cubierta en los ecosistemas semiáridos con imágenes hiperespectrales, caso práctico en el Parque Natural Cabo de Gata-Níjar (Almería). Ecosistemas 17:79-88

García D. 2006. La escala y su importancia en el análisis espacial. Ecosistemas 15:7-18.

Guo Q. y Brown J.H. 1996. Temporal fluctuations and experiment effects in desert plant communities. Oecologia 107:568-577

Hill M.O. 1979. TWINSPAN: a Fortran Program for Arranging Multivariate Data in an Ordered Two-Way Table by Classification of the Individuals and Attributes. Section of Ecology and Systematics, Cornell University.

Hutchings M.J. y de Kroon H. 1994. Foraging in plants: the role 
of morphological plasticity in resource acquisition. Advances in Ecological Research 25:159-238.

Jongman R.H., ter Braak C.J.F. y van Tongeren O.F.R. 1987. Data Analysis in Community and Landscape Ecology. Pudoc Wageningen.

Lieberman D., Lieberman M., Peralta R. y Hartshorn G.S. 1996. Tropical forest structure and composition on a large-scale altitudinal gradient in Costa Rica. Journal of Ecology 84:137-152.

Lindenmayer D.B., Margules C.R. y Botkin D.B. 2000. Indicators of Biodiversity for Ecologically Sustainable Forest Management. Conservation Biology 14: 941-950.

Louman B., Valerio J. y Jiménez W. 2001. Bases ecológicas. En: Louman B., Quirós D. y Nilsson M. Eds. 2001. Silvicultura de bosques latifoliados húmedos con énfasis en América Central, pp.21-80. CATIE. Turrialba, Costa Rica.

McCune B. y Mefford M.J. 2006. PC-ORD. Multivariate analysis of ecological Data V.5.10. MjM Software Design Gleneden Beach, Oregon.

Meave J., Carabias J., Arriaga V. y Valiente-Banuet A. 1994. Observaciones fenológicas en el Pedregal de San Ángel. En: Rojo A. Ed. Reserva ecológica el Pedregal de San Ángel: ecología, historia natural y manejo, pp.91-105, Universidad Nacional Autónoma de México. México, D.F.

Menge B.A., Berlow E.L., Blanchette C.A., Navarrete S.A. y Yamada S.B. 1994. The keystone species concept: Variation in interaction strength in a rocky intertidal habitat. Ecological Monographs 64:250-286.

Paruelo J.M., Piñeiro G., Oyonarte C., Alcaraz D., Cabello J. y Escribano P. 2005. Temporal and spatial patterns of ecosystem functioning in protected arid areas of southeastern Spain. Applied Vegetation Science 8:93-102.

Peralta-Higuera A. y Prado-Molina J. 2009. Los límites y la cartografía. En: Lot A. y Cano-Santana Z. Eds. Biodiversidad del ecosistema del Pedregal de San Ángel pp.27-42, Universidad Nacional Autónoma de México. México, D.F.

Pickett S.T.A. y Rogers K.H. 1997. Patch dynamics: the transformation of landscape structure and function. En: Bisonette J.A. Ed. Wildlife and Landscape Ecology: Effects of Pattern and Scale, pp.101-127, Springer-Verlag, New York.

Rosenberg D.B., Freedman S.M. 1994. Temporal heterogeneity and ecological community structure. International Journal of Environmental Studies 46:97-102.

Root R.B. 1967. The niche exploitation pattern of the blue-grey gnatcatcher. Ecological Monographs 37:317-350

Rzedowski J. 1954. Vegetación del Pedregal de San Ángel (Distrito Federal, México). Anales de la Escuela Nacional de Ciencias Biológicas 8:59-130.

Santibáñez-Andrade G. 2005. Caracterización de la heterogeneidad ambiental en la Reserva del Pedregal de San Ángel. Tesis de Licenciatura, Facultad de Ciencias, Universidad Nacional Autónoma de México. México, D.F. 67 pp.

Shiyomi M. y Yoshimura J. 2000. Measures of spatial heterogeneity for species occurrence or disease incidence with finitecounts. Ecological Research 15:13-20.

Simberloff D. 1998. Flagship, umbrellas, and keystones: is singlespecies management passé in the landscape era? Biological Conservation 83:247-257.

Smith, R.L. y Smith T.M. 2001. Ecología. Pearson Education, Madrid, España.

SPSS Inc. 2003. SPSS 12.0 for Windows. SPSS Inc. Chicago Il.

Statsoft Inc. 2001. Statistica for Windows. Manual version 6.0. Statsoft Inc. Tulsa, Oklahoma.

Stewart A.J.A., Jonh E.A. y Hutchings M.J. 2000. The world is heterogeneous: ecological consequences of living in a patchy environment. En: Hutchings M.J., John E.A., Stewart A.J.A. 2000. The Ecological Consequences of Environmental Heterogeneity, pp.1-8, Blackwell Science Ltd. Oxford.

Swaine M.D. y Whitmore T.C. 1988. On the definition of ecological species groups in tropical rain forest. Vegetatio 75:81-86.

Tanner J.E., Hughes T.P. y Connell J.H. 1994. Species coexistence, keystone species, and succession: A sensitivity analysis. Ecology 75:2204-2219.

Tatsuoka M.M. 1971. Multivariate analysis. John Wiley \& Sons Inc. New York.

Titus J.H. y Tsuyuzaki S. 2003. Distribution of plants in relation to microsites on recent volcanic substrates on Mount Koma, Hokkaido, Japan. Ecological Research 18:91-98.

Tongway D.J., Cortina J. y Maestre F.T. 2004. Heterogeneidad espacial y gestión de medios semiáridos. Ecosistemas 13:2-15.

Wiens J.A. 1989. Spatial scaling in ecology. Functional Ecology 3:385-397.

Xu M., Qi Y., Chen J. y Yin W. 2000. Effects of spatial heterogeneity of microenvironment on plant biodiversity in the Southeastern Missouri Ozarks. Geographic Information Sciences 61:38-49.

Recibido: 23 de abril de 2009

Aceptado: 29 de septiembre de 2009 